\title{
CO-CREATION: CONCEPTUALIZATION AND RESEARCH IMPLICATIONS
}

\author{
Kumar Rakesh Ranjan, Indian Institute of Management, India \\ Shainesh G., Indian Institute of Management, India
}

\begin{abstract}
Research in the domain of value co-creation has progressed extensively over the last twelve years, but the conceptualization of value co-creation is still equivocal. We try to fill this important gap through a systematic review of the co-creation research that appeared in leading Marketing journals during the period 2000-2013. We used the keyword search for the word cocreation and its different variations in the ABI/INFORMS - Proquest and Business Source Complete (EBSCO) databases. After initial round of screening, we reviewed around 175 papers.

The review is organized into three major sections. The first section discusses the progression of the field - for example, the dominant contributors to the field, the key domains of application of co-creation concept across firms and consumers, the major research methods, research context, and sample characteristics. We conclude this section by highlighting the allied and emerging areas of application of the construct and by subsequently trying to map the potential growth of the field. In the second section we systematically analyzed the content on co-creation that was created from the reviewed papers. We create a complex typology of constructs and their adjacent labels and classify them as the antecedent, consequents, mediators, and moderators in the nomological net of co-creation. Additionally, we are able to explicate the concept of co-creation and offer a conceptual framework for the process of co-creation. The third section concludes this review by problematizing some of the assumption and pluralities in the understanding of co-creation. We offer four areas of novel research inquiries - one each in the area of process of co-creation, the phenomenon of exploitation of consumers, the over-emphasis of consumer interaction and the arising need for non-traditional organizational forms, and the related issue of skill and capabilities of consumers.
\end{abstract}

References available upon request 